\title{
Lymphoscintigraphy Using Dynamic Imaging and SPECT/CT in Chylothorax
}

\author{
Asami Ohtsuka ${ }^{1}$, Yusuke Inoue ${ }^{1}$, Yuji Asano ${ }^{1}$, Reiko Woodhams ${ }^{1}$, Kazu Shiomi $^{2}$ \\ ${ }^{1}$ Department of Diagnostic Radiology, Kitasato University School of Medicine, Sagamihara, Japan \\ ${ }^{2}$ Department of Thoracic Surgery, Kitasato University School of Medicine, Sagamihara, Japan \\ Email: inoueys34@gmail.com
}

Received June 1, 2013; revised June 30, 2013; accepted July 14, 2013

Copyright (C) 2013 Asami Ohtsuka et al. This is an open access article distributed under the Creative Commons Attribution License, which permits unrestricted use, distribution, and reproduction in any medium, provided the original work is properly cited.

\begin{abstract}
Lymphoscintigraphy readily provides confirmation of chylothorax but not detailed localization of the leakage site. A 77-year-old woman developed traumatic chylothorax and underwent lymphoscintigraphy with radiolabeled albumin to identify the site of lymph leakage. Dynamic imaging demonstrated appearance of focal activity presumably in the upper mediastinum, followed by spread to the left hemithorax. Subsequently, SPECT/CT showed that the leakage site was located in the left side of the upper mediastinum, and this location was confirmed during video-assisted thoracoscopic surgery. Dynamic imaging demonstrated the site of first appearance of abnormal activity, and SPECT/CT enabled detailed localization of the abnormal activity with anatomic correlation. The combination of dynamic imaging with SPECT/CT appears to be recommendable for lymphoscintigraphic assessment of chylothorax.
\end{abstract}

Keywords: Chylothorax; Lymphoscintigraphy; ${ }^{99 m}$ Tc-DTPA-HSA; Dynamic Imaging; SPECT/CT

\section{Introduction}

Chylothorax is defined as accumulation of lymph fluid in the pleural space, and may occur due to traumatic, neoplastic, and idiopathic causes [1,2]. It is usually treated conservatively by drainage of chylous pleural effusion and fasting. When conservative treatment is insufficient, surgical treatment may be chosen. Lymphoscintigraphy has been applied for preoperative evaluation of chylothorax to confirm the diagnosis of chylothorax and to assess the site of lymph leakage [3-9]. We report a patient with traumatic chylothorax in whom preoperative localization of the leakage site was attained by lymphoscintigraphy using dynamic imaging and single photon emission computed tomography/computed tomography (SPECT/CT).

\section{Case Report}

A 77-year-old woman stabbed herself in the left side of the neck with a kitchen knife and was admitted to our emergency center. She had senile depression and had attempted suicides several times. On admission, her consciousness was disturbed. Her maximal blood pressure was $60 \mathrm{mmHg}$, her pulse rate was $100 / \mathrm{min}$, and her respiratory rate was $30 / \mathrm{min}$. Her blood count and biochemistry levels were found to be within normal ranges. Con- trast-enhanced CT of the chest revealed bleeding from the left common carotid artery at the level of the left clavicle and left hemopneumothorax. Subsequently, a covered stent was inserted into the left common carotid artery, and a chest tube for drainage was inserted into the left thoracic cavity. The drained fluid was bloody.

She fasted from admission, and enteral nutrition was started on the second day of hospitalization, followed by a gradual increase in the amount of intake. On the sixth day, fluid drained from the chest tube changed to a milky-white color, indicating development of chylothorax. Although enteral nutrition was stopped, the chest drain output was more than $1500 \mathrm{~mL} /$ day and surgical treatment was planned.

Lymphoscintigraphy was performed on the eighth day to identify the site of lymph leakage before surgery. After subcutaneous injection of ${ }^{99 \mathrm{~m}}$ Tc-diethylenetriaminepentaacetic acid human serum albumin $\left({ }^{99 m}\right.$ Tc-DTPAHSA, $185 \mathrm{MBq} / 0.5 \mathrm{~mL}$ ) into the dorsum of each foot, anterior and posterior dynamic images of the chest were obtained at a rate of 1 frame per min for $40 \mathrm{~min}$. An SPECT/CT system (Symbia T2; Siemens, Erlangen, Germany) equipped with a low-energy high-resolution collimator was used. At 12 min after injection, focal activity appeared near the center of the upper chest, slightly shifted to the left (Figure 1). Thereafter, activity spread 


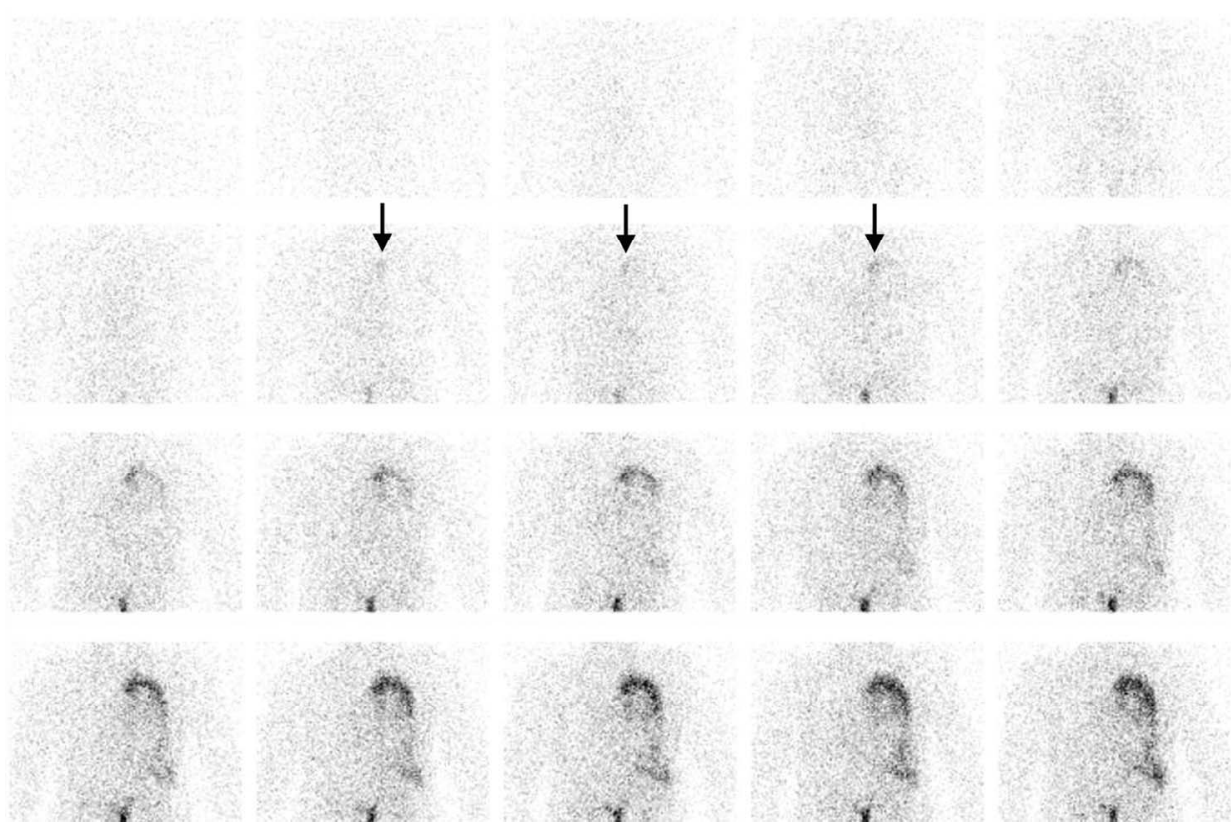

Figure 1. Anterior dynamic images of the chest after subcutaneous injection of ${ }^{99 \mathrm{~m}}$ Tc-DTPA-HSA into both feet. The images were reframed to produce 20 2-min images. At $12 \mathrm{~min}$ after injection, focal activity appeared presumably in the upper mediastinum (arrows), followed by spread to the left side.

gradually to the left side. These findings indicated leakage at the left side of the upper mediastinum due to disruption of the thoracic duct and influx into the left thoracic cavity. For detailed localization, SPECT/CT was performed immediately after the completion of dynamic imaging. It demonstrated that the first appearance site of focal activity on dynamic imaging was located in the left side of the upper mediastinum, just above the aortic arch, and that radioactive materials was distributed in the left thoracic cavity (Figure 2).

Video-assisted thoracoscopic surgery was performed on the 10th day to ligate the thoracic duct. Milk was administered into the intestine before the surgery, and leakage from the thoracic duct was confirmed during surgery at the site indicated by lymphoscintigraphy (Figure 3 ). The thoracic duct was ligated near the leakage site. After the surgery, the amount of chylous fluid drained from the chest tube markedly decreased. A low-fat diet was started on the 10th postoperative day, and her physical and mental condition improved.

\section{Discussion}

The patient presented here was found to have hemopneumothorax on admission and developed chylothorax 6 days later. It is inferred that the cause of chylous leakage was penetrating trauma with a kitchen knife and that fasting had prevented evident leakage of lymph fluid into the pleural cavity initially. Once massive leakage occurred, it was not well controlled conservatively and was treated surgically.

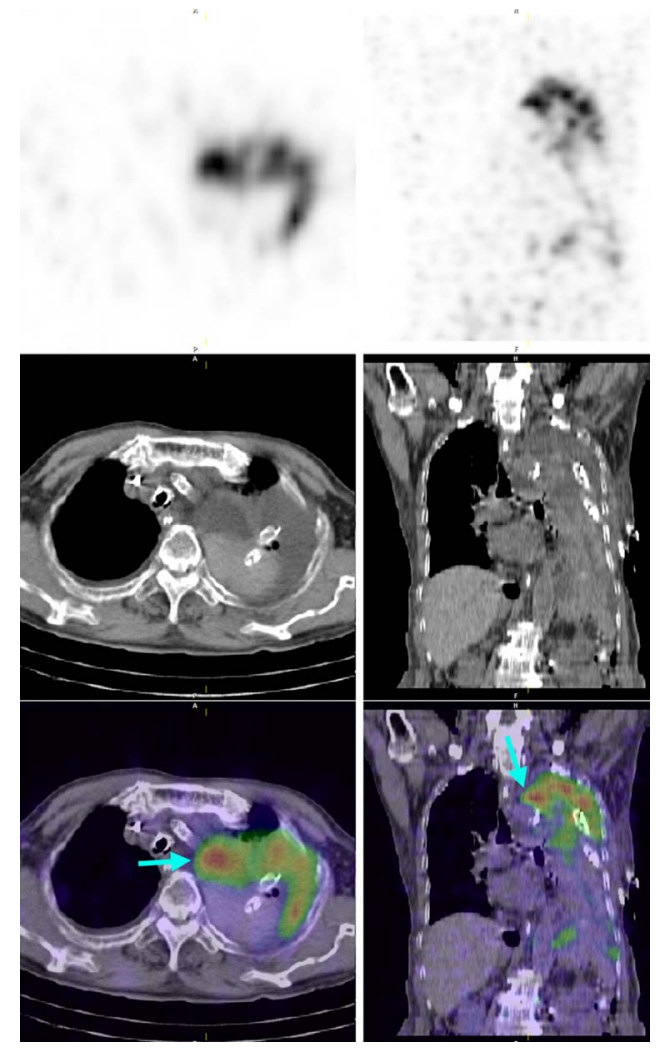

Figure 2. Axial (left) and coronal (right) SPECT/CT images. From top to bottom, SPECT images, plain CT images, and SPECT/CT fusion images are presented. Intense radioactivity is shown at the left side of the upper mediastinum (arrows), just above the calcified aortic arch, extending to the left thoracic cavity. 


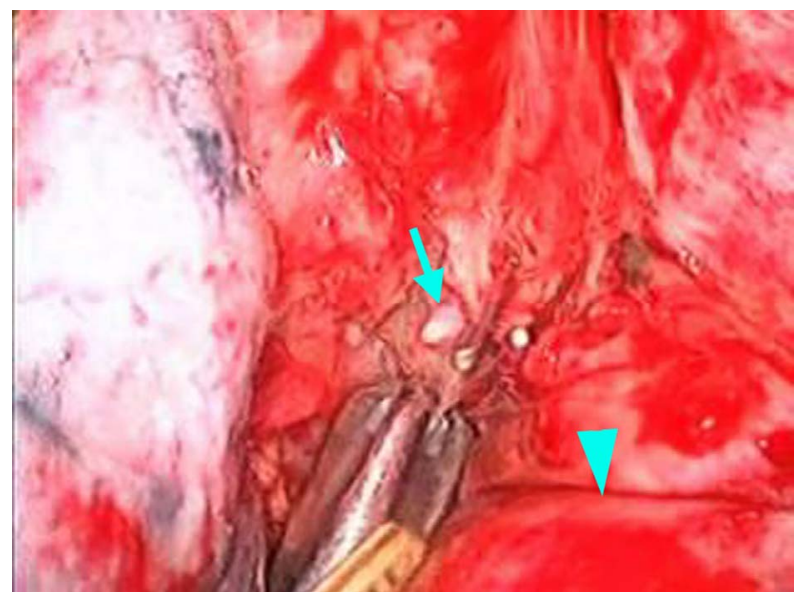

Figure 3. Thoracoscopic image during surgery. Leakage of milk is evident (arrow) just above the aortic arch (arrowhead).

Although X-ray lymphangiography allows definitive diagnosis of chylothorax and accurate localization of leakage $[1,10]$, the procedure is rather complicated because of the need for direct injection of contrast materials into the lymph vessels. Lymphoscintigraphy is a convenient alternative to lymphangiography. Radiotracers are injected subcutaneously, and then enter the lymphatic vessels spontaneously. Radiocolloids are trapped effectively by the lymph nodes and are frequently used for lymphoscintigraphy. Although they have also been applied to evaluation of chylothorax [3,9], radiolabled albumin is cleared from the injection site more rapidly than radiocolloids [11] and appears to be suitable for evaluation of lymph leakage [4-8].

In the patient presented here, lymphoscintigraphy with ${ }^{99 \mathrm{~m}}$ Tc-DTPA-HSA was performed to identify the site of leakage preoperatively. First, dynamic planar imaging of the chest was performed, and focal activity was first detected presumably in the left side of the upper mediastinum. Subsequent SPECT/CT confirmed that the site where focal activity first appeared was the left side of the upper mediastinum and demonstrated that the site was located just above the aortic arch. Video-assisted thoracoscopic surgery was performed using this information as a guide, and intraoperative observation proved leakage of milk administered into the intestine at the site indicated by lymphoscintigraphy.

SPECT/CT enables detailed localization of abnormal activity with anatomic correlation and has been applied to lymphoscintigraphic assessment of chylothorax [7-9]. Our findings are consistent with the previous reports in terms of the usefulness of SPECT/CT. Additionally, the role of dynamic imaging appears to be worth emphasizing [4]. Repeated acquisition of static images with some intervals is commonly performed in lymphoscintigraphy, and the imaging may start $30 \mathrm{~min}$ after tracer injection or later. It appears to be sufficient for definitive diagnosis of chylothorax but not for localization of leakage. After the tracer leaks from lymph vessels, it will move from the leakage site and may spread widely before the start of imaging or during intervals of imaging. SPECT/CT allows demonstration of the distribution of radioactivity at the time point of data acquisition; however, the wide spread may disturb identification of the actual leakage site. Dynamic imaging provides the time course of tracer distribution and appears to facilitate recognizing the site of first appearance of abnormal activity, as illustrated in our patient. The combination of dynamic imaging with SPECT/CT is recommendable for the assessment of chylothorax.

\section{REFERENCES}

[1] I. E. Platis and C. E. Nwogu, "Chylothorax,” Thoracic Surgery Clinics, Vol. 16, No. 3, 2006, pp. 209-214. doi:10.1016/j.thorsurg.2006.05.011

[2] V. G. Valentine and T. A. Raffin, "The Management of Chylothorax,” Chest, Vol. 102, No. 2, 1992, pp. 586-591. doi:10.1378/chest.102.2.586

[3] S. Heyman and R. Wilmott, "Chylothorax on Technetium-99m Antimony Sulfide Colloid Scan,” Journal of Nuclear Medicine, Vol. 27, No. 11, 1986, pp. 1783-1784.

[4] Y. Inoue, T. Otake, J. Nishikawa and Y. Sasaki, "Lymphoscintigraphy Using Tc-99m Human Serum Albumin in Chylothorax," Clinical Nuclear Medicine, Vol. 22, No. 1, 1997, p. 60. doi:10.1097/00003072-199701000-00018

[5] K. Suga, N. Kume, A. Hara, G. Miura, N. Matsunaga, K. Sugi, et al., “Abnormal Lymphatic Flow Demonstrated by Lymphoscintigraphy in Chylothorax Correlation with Lymphography,” Clinical Nuclear Medicine, Vol. 24, No. 9, 1999, pp. 716-717. doi:10.1097/00003072-199909000-00026

[6] T. Stavngaard, J. Mortensen, J. Brenoe and L. B. Svendsen, "Lymphoscintigraphy Using Technetium-99m Human Serum Albumin in Chylothorax," The Thoracic and Cardiovascular Surgeon, Vol. 50, No. 4, 2002, pp. 250252. doi:10.1055/s-2002-33099

[7] M. Momose, S. Kawakami, T. Koizumi, K. Yoshida, S. Kanda and R. Kondo, et al., "Lymphoscintigraphy Using Technetium-99m HSA-DTPA with SPECT/CT in Chylothorax after Childbirth,” Radiation Medicine, Vol. 26, No. 8, 2008, pp. 508-511.

doi:10.1007/s11604-008-0265-4

[8] D. Kayano, J. Taki, H. Wakabayashi and S. Kinuya, “Tc99m Human Serum Albumin Lymphoscintigraphy with SPECT/CT in Chylothorax," Clinical Nuclear Medicine, Vol. 36, No. 11, 2011, pp. 1056-1057. doi:10.1097/RLU.0b013e31821a2ba3

[9] N. Prevot, O. Tiffet, J. Avet Jr., E. Quak, M. Decousus and F. Dubois, "Lymphoscintigraphy and SPECT/CT Using ${ }^{99 \mathrm{~m}}$ Tc Filtered Sulphur Colloid in Chylothorax," European Journal of Nuclear Medicine and Molecular Imaging, Vol. 38, No. 9, 2011, p. 1746. 
doi:10.1007/s00259-011-1793-1

[10] D. J. Boffa, M. J. Sands, T. W. Rice, S. C. Murthy, D. P. Mason, M. A. Geisinger, et al., "A Critical Evaluation of a Percutaneous Diagnostic and Treatment Strategy for Chylothorax after Thoracic Surgery,” European Journal of Cardio-Thoracic Surgery, Vol. 33, No. 3, 2008, pp.
435-439. doi:10.1016/j.ejcts.2007.11.028

[11] E. Ohtake, K. Matsui, Y. Kobayashi and Y. Ono, “Dynamic Lymphoscintigraphy with Tc-99m Human Serum Albumin,” Radiation Medicine, Vol. 1, No. 2, 1983, pp. 132-136. 